Urologe 2009 · 48:1427-1428

DOI 10.1007/s00120-009-2154-6

Online publiziert: 9. Dezember 2009

(c) Springer Medizin Verlag 2009

\author{
M. Giessing ${ }^{1} \cdot$ K. Dreikorn ${ }^{2}$ \\ ${ }^{1}$ Urologische Klinik, Heinrich-Heine-Universitätsklinikum Düsseldorf \\ ${ }^{2}$ Bremen
}

\title{
Nierentransplantation: Quo vadis?
}

Die Transplanteure finden sich heuDie Nierentransplantation (NTX) wurde in den letzten Jahrzehnten weitestgehend standardisiert und stellt heute sowohl bei Erwachsenen als auch bei Kindern ein etabliertes und bei den meisten Patienten grundsätzlich das anzustrebende Therapieverfahren der chronischen Niereninsuffizienz dar. Hauptgründe hierfür sind die im Vergleich zur Dialysebehandlung höheren Überlebensraten und besseren Rehabilitationsmöglichkeiten. In Abhängigkeit vom Alter und evtl. bestehenden Komorbiditäten des Empfängers können heute bei erwachsenen Transplantatempfängern 5-Jahres-Patientenüberlebensraten von $>80 \%$ erreicht werden, mit 5-Jahres-Transplantatfunktionsraten von $>70 \%$ nach der Transplantation von Nieren verstorbener Spender und von $>85 \%$ nach der Transplantation von Lebendspendernieren.

Aktuell wird der operative Eingriff der Transplantation in Deutschland an $40 \mathrm{Kli}$ niken durchgeführt. In ca. der Hälfte dieser Zentren erfolgt die NTX durch die Urologen selber oder in Kooperation von Urologie und Chirurgie.

Heute, nach mehr als 40 Jahren NTX in Deutschland mit $>62.000$ durchgeführten Nierentransplantationen, stehen wir an einem Scheideweg. Die Pioniere und langjährigen Förderer der Nierentransplantation sind mittlerweile auf diesem Gebiet nicht mehr aktiv tätig oder stehen kurz vor dem Ruhestand - eine neue Generation übernimmt die Transplantationsmedizin. Wird es ihr gelingen, diesen so wertvollen und interessanten Teil der Medizin mit dem erforderlichen „Herzblut“ und nicht nur mit DRG-Optimierungsstrategien zu erfüllen? te mit einem deutlich älteren und morbideren Patientengut konfrontiert als noch vor 10 Jahren. Dies betrifft einerseits die Spender, die zu mehr als einem Drittel $>65$ Jahre alt sind und auch aus anderen Gründen noch vor 15 Jahren nicht als Spender akzeptiert wurden. Aber auch die Empfänger werden älter, und mit dem Empfängeralter steigen die Anforderungen an die Transplantationsmediterdisziplinäre Kooperation aller bei der NTX involvierten medizinischen Fachbereiche das Gebot der Stunde und der Zukunft. Urologen, Nephrologen, Gefäßund Transplantationschirurgen, aber auch Radiologen, Immunologen, Psychologen, Soziologen, bei der Diagnostik tätige $\mathrm{Pa}-$ thologen und pädiatrische Nephrologen (bei der Transplantation von Kindern) sollten eng zusammenarbeiten, um langfristig optimale Ergebnisse für den Empfänger - und im Falle der Lebendnierenspende auch für den Spender - zu gewährleisten. Somit sollten auch die Zeiten, in denen bei der Neubesetzung eines urologischen Lehrstuhls dessen Transplantationsaktivität oft automatisch und komplett an die Chirurgie übergeht, endgültig der Vergangenheit angehören und vielmehr die gemeinsame urologisch-chirurgische Expertise im Vordergrund stehen, zum Wohle der Patienten.

So hat z. B. auch die von den Urologen initiierte und weiterentwickelte laparoskopische Donornephrektomie beim Lebendspender als minimal-invasive Operationsmethode das operative Repertoire wesentlich erweitert.

Der Urologe ist sowohl bei der Auswahl und Vorbereitung der Patienten für eine zin. Schon aus diesen Gründen ist die in-
Transplantation, als auch bei der Spendernierenentnahme und der Diagnostik und Therapie urologischer Komplikationen und Erkrankungen ein unverzichtbarer Kooperationspartner. Leider ist das Problem des persistierenden Mangels an (postmortalen) Spenderorganen seit Jahrzehnten weiterhin ungelöst geblieben.

Auch das 1997 eingeführte Transplantationsgesetz hat zu keiner Verbesserung geführt. Die Wartezeiten für den zur Transplantation angemeldeten Patienten, bis eine geeignete postmortale Spenderniere zur Verfügung steht, liegen inzwischen bei 7-8 Jahren. Den im Jahre 2008 in Deutschland durchgeführten 2188 Nierentransplantationen mit postmortalen Spenderorganen stehen 8000 Patienten auf der Warteliste gegenüber.

Im Vergleich zu anderen Ländern weist Deutschland mit 16 postmortalen Organspendern/Mio. Einwohner (E.) ein erhebliches Defizit auf. So liegt die Rate z. B. in Spanien bei 34 Organspendern/Mio. E. und kommt damit der berechneten Rate von 35-50 möglichen postmortalen Organspendern/Mio. E. sehr viel näher.

Auch wenn die Ergebnisse der Transplantation von Nieren lebender Spender besser sind als bei der Verwendung von postmortalen Spendern und somit dem Empfänger viele Vorteile bringen, darf nicht übersehen werden, dass sich ein Lebendspender aus altruistischen Gründen einer für ihn nicht ohne potentielle Risiken behafteten Operation unterzieht, auch wenn ernsthafte Komplikationen selten geworden sind.

Im Jahre 2008 wurden 565 Lebendspendernieren transplantiert, entsprechend einem Anteil von 20,5\% an der Anzahl aller durchgeführten Nierentrans- 
plantationen, mit ansteigender Tendenz. Vergleichsweise sind Lebendspendertransplantationen in Spanien selten, da die meisten Dialysepatienten dort innerhalb eines Jahres mit Nieren verstorbener Spender transplantiert werden können. Ob eine Novellierung des Transplantationsgesetzes (z. B. mit Einführung der Widerspruchslösung) zu einem Anstieg postmortaler Organspender führen könnte, wird derzeit sehr kontrovers diskutiert und erscheint $\mathrm{u}$. E. eher fraglich.

Bemerkenswert und ebenfalls kritisch zu diskutieren erscheint das Vorgehen der Ärztekammer Mecklenburg-Vorpommern: als vor einigen Jahren festgestellt wurde, dass 3 Kliniken des Landes nicht die im Gesetz vorgeschriebene Meldung potentieller Organspender vornahmen und somit keine Organentnahmen durchgeführt wurden, drohte die Ärztekammer der jeweiligen Klinikleitung mit dem Entzug der Weiterbildungserlaubnis für ihr Haus. „Kaum waren die Briefe draußen“, erinnert sich Ärztekammer Präsident Dr. A. Crusius, „wurden die ersten Spender gemeldet.“

Ob hiermit eine dauerhafte Steigerung der Organspende erzielt werden kann, bleibt abzuwarten, denn die mit der Organspende verbundenen Anforderungen an sog. Spenderkrankenhäuser sollten nicht unterschätzt werden. „Zurückhaltende“ Einstellungen zur Organspende sollten angesichts der finanziellen und personellen Ressourcenlimitierungen den Krankenhäusern nicht kritiklos vorgeworfen sondern konstruktiv diskutiert werden. „Zwangsmaßnahmen“ erscheinen eher problematisch.

Die Vorreiterrolle Spaniens auf dem Gebiete der Organspende liegt wohl eher an strukturellen und logistischen Besonderheiten mit adäquater Professionalisierung und finanzieller Unterstützung der Organspende in den Krankenhäusern. Wir sollten aus dem „spanischen Modell“ lernen, die Vorteile in Deutschland umsetzen den bisher unwirksamen Slogan "Organspende ist eine gemeinschaftliche Aufgabe“ endlich mit Leben füllen.

Im vorliegenden Heft beleuchten Kollegen aus einigen urologischen Nierentransplantationszentren aktuelle und interessante Facetten der Transplantationsmedizin. Als „Highlight“ haben wir Fr.
Prof. Dr. med. G. Offner, Fr. Dr. med. U. John und Frau Dr. med. M. Oldhafer, die als pädiatrische Nephrologen bzw. Kinder- und Jugendmediziner unserem Arbeitskreis besonders nahe stehen, dafür gewinnen können, über ihre Erfahrungen bei der Nierentransplantation im Kindesund Jugendalter zu berichten.

Engagement auf dem Gebiete der Nierentransplantation bietet der Urologin und dem Urologen ein interessantes, vielfältiges, dankbares, sowohl wissenschaftlich als auch klinisch attraktives und operativ herausforderndes (urologisches!) Betätigungsfeld und stärkt die Bedeutung der Urologie im Zusammenspiel der verschiedenen medizinischen Fachgebiete.

Der Arbeitskreis Nierentransplantation sieht eine seiner Hauptaufgaben darin, das Gebiet der Nierentransplantation für das urologische Fach zu erhalten und interessierte jüngere Kolleginnen und Kollegen für die interdisziplinäre Kooperation in diesem Bereich zu motivieren und zu begeistern.

Der Vorstand des Arbeitskreises dankt den Herausgebern für die Möglichkeit, seinen Lesern einen Einblick einige interessante Aspekte der Nierentransplantation zu vermitteln.

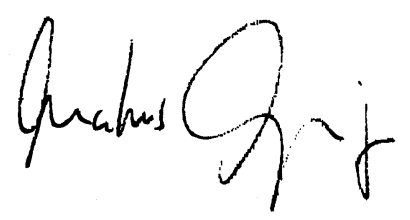

M. Giessing

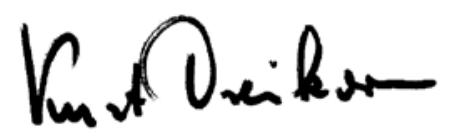

\section{K. Dreikorn}

Für den Arbeitskreis Nierentransplantation der Deutschen Gesellschaft für Urologie

Akademie der Deutschen Urologen

\section{Korrespondenzadresse \\ PD Dr. M. Giessing}

Urologische Klinik,

Heinrich-Heine-Universitätsklinikum,

Moorenstraße 5, 40225 Düsseldorf

Markus.Giessing@med.uni-duesseldorf.de

\section{Premiere des Interdisziplinären Beckenbodenkongresses}

Viele Gründe können für Erkrankungen des Beckenbodens und der Beckenbodenorgane verantwortlich sein. Eine interdisziplinäre Therapie stellt eine effektive Behandlung im Sinne des Patienten dar. Vor diesem Hintergrund veranstaltete das Neue Deutsche Interdisziplinäre Beckenbodenzentrum am 16./17. Oktober 2009 in Berlin erstmals den 1. Interdisziplinären Beckenbodenkongress (IBK) gemeinsam mit dem Universitätsklinikum Jena und der Arbeitsgemeinschaft für Urogynäkologie und plastische Beckenbodenrekonstruktion e.V. (AGUB) und der Deutschen Gesellschaft für Gynäkologie und Geburtshilfe e.V. (DGGG). Die neuen Behandlungsformen bei Harn- und Analinkontinenz von Frau und Mann waren Schwerpunkt der Veranstaltung. Interaktive Workshops und Live-Operationen per Videositzungen demonstrierten innovative Therapieformen. Der gemeinsame Austausch zu den neuesten medizinischen Erkenntnissen und angewandten Therapieverfahren der verschiedenen Fachbereiche wurde intensiv genutzt, um für die Zukunft den Patienten bessere Behandlungsoptionen für mehr Lebensqualität anzubieten.

Quelle: Neues Deutsches Interdisziplinäres Beckenbodenzentrum, www. neues-deutsches-beckenbodenzentrum.de 